\title{
EFECTO DE DIVERSAS ACTIVIDADES FÍSICAS Y DEPORTIVAS SOBRE LA AUTOEFICACIA DE ESTUDIANTES UNIVERSITARIOS COSTARRICENSES
}

THE EFFECT OF DIFFERENT SPORT AND PHYSICAL ACTIVITY COURSES ON SELFEFFICACY OF COSTA RICAN UNIVERSITY STUDENTS

\section{Volumen 13, Número 3 \\ Setiembre - Diciembre}

pp. 1-17

Este número se publicó el 30 de setiembre de 2013

Cinthya Campos Salazar

Andrea Solera Herrera

Revista indizada en REDALYC, $\underline{\text { SCIELO }}$

Revista distribuida en las bases de datos:

CATÁLOGO DE LATINDEX, IRESIE, CLASE, DIALNET, DOAJ, E-REVIST@S, SHERPA/ROMEO, QUALIS, MIAR

Revista registrada en los directorios:

ULRICH'S $, \underline{R E D I E}, \underline{R I N A C E}, \underline{\text { OEI }}, \underline{\text { MAESTROTECA }}, \underline{\text { PREAL, }} \underline{\text { CLASCO }}$ 


\title{
EFECTO DE DIVERSAS ACTIVIDADES FÍSICAS Y DEPORTIVAS SOBRE LA AUTOEFICACIA DE ESTUDIANTES UNIVERSITARIOS COSTARRICENSES
}

\author{
THE EFFECT OF DIFFERENT SPORT AND PHYSICAL ACTIVITY COURSES ON SELF- \\ EFFICACY OF COSTA RICAN UNIVERSITY STUDENTS
}

\author{
Cinthya Campos Salazar ${ }^{1}$ \\ Andrea Solera Herrera²
}

\begin{abstract}
Resumen: El presente artículo fue realizado con estudiantes universitarios matriculados en el curso de "Actividad Deportiva" de la Universidad de Costa Rica. Los propósitos fueron: (1) comprobar si existe relación significativa entre el nivel de autoeficacia, entendida como la confianza en sí mismo para iniciar y mantenerse en un plan de ejercicio, y la cantidad de actividad física que reporta el estudiantado al inicio del curso; (2) establecer si existe un efecto significativo de las diversas actividades físicas y deportivas en el nivel de autoeficacia, tanto para efectuar ejercicio como para no abandonarlo; y (3) comprobar si los cambios en la autoeficacia son influenciados por el género o el tipo de actividad física realizada (cursos de acondicionamiento físico versus cursos de deportes). Se trabajó con 211 estudiantes matriculados en el III ciclo de 2009, durante el verano. Al estudiantado se le aplicó, antes de iniciar el curso y una vez finalizado, la encuesta de autoeficacia para comportamiento de ejercicio y el Cuestionario Internacional de Actividad Física (IPAQ), con el fin de medir el nivel de actividad física. Los resultados mostraron una asociación positiva entre el nivel de actividad física reportada por el(la) estudiante y el nivel de autoeficacia $(p<0.001)$. En cuanto a este último, las mujeres presentaron niveles inferiores que los varones $(p<0.001)$, asimismo el nivel de autoeficacia aumentó significativamente $(p<0.05)$ independientemente del género y del tipo de actividad física. En conclusión, los cursos de actividad deportiva produjeron efectos positivos en la autoeficacia de los participantes, además, los estudiantes más activos presentaron mayores niveles de ella.
\end{abstract}

Palabras clave: AUTOEFICACIA, ESTUDIANTES UNIVERSITARIOS, ACTIVIDAD FÍSICA, ACTIVIDAD DEPORTIVA, UNIVERSIDAD DE COSTA RICA

\begin{abstract}
This article was conducted with college students enrolled in the course "Sports Activity" at the University of Costa Rica. The purposes were: (1) to determine whether significant associations existed between levels of self-efficacy, defined as the confidence to begin and stay on an exercise program, and the amount of physical activity reported by the students at the beginning of the course; (2) to determine whether significant effects of selected physical and sport activities on self-efficacy levels exist, both, for performing exercise and for avoiding dropping off exercise; and (3), to test whether changes in self-efficacy are influenced by gender or type of physical activity (fitness courses versus sport courses). Participants were 211 students registered in the third session term 2009, during Costa Rican summer. Students responded at the beginning and at the end of the course the selfefficacy survey for exercise behavior and the International Physical Activity Questionnaire (IPAQ) in order to measure the levels of physical activity. The results indicated a positive association between the level of physical activity reported by students and self-efficacy $(p<0.001)$. Females showed lower levels of self-efficacy than males $(p<0.001)$ and the level of self-efficacy increased significantly $(p<0.05)$ regardless of gender and physical activity. In conclusion, most active students showed higher self-efficacy scores and sport courses elicited positive effects on participants' self-efficacy.
\end{abstract}

Key words: SELF-EFFICACY, COLLEGE STUDENTS, PHYSICAL ACTIVITY, SPORT, UNIVERSITY OF COSTA RICA

\footnotetext{
${ }^{1}$ Profesora de la Escuela de Educación Física y Deportes de la Universidad de Costa Rica. Maestría en Educación con énfasis en Educación Física, de la Universidad de Costa Rica. Dirección electrónica: cinthia.campos@ucr.ac.cr

2 Profesora de la Escuela de Educación Física y Deportes de la Universidad de Costa Rica. Ph.D. en Ciencias del Ejercicio, de la Universidad Alemana del Deporte, Colonia, Alemania.Dirección electrónica: andrea.solera@ucr.ac.cr
}

Artículo recibido: 6 de marzo, 2013

Aprobado: 12 de setiembre, 2013 


\section{Introducción}

Actualmente, es contundente el beneficio del ejercicio y la actividad física en los distintos sistemas corporales. Durante los últimos años, se han incrementado las investigaciones que relacionan la actividad física con componentes psicológicos que se asocian al bienestar general del individuo. Dos estudios realizados en la Universidad de Costa Rica han comprobado que participar en un curso de actividad física o deporte produce beneficios sobre el estado de ánimo del estudiantado (Campos y Moncada, 2009 y 2012), estos resultados lo confirman Legrand y Phillipe (2007) y Rethorst, Wipfli y Landers (2009), quienes reportan, en sus meta-análisis, un beneficio del ejercicio sobre los niveles de depresión. Sin embargo, tomar la decisión de iniciar un plan de ejercicios o mantenerse en él es un factor que requiere de mayor indagación. Una de las formas en que se ha abordado el tema es por medio del estudio de la autoeficacia y su relación con la actividad física.

La autoeficacia, como constructo psicológico, ha sido ampliamente estudiada (Bandura, 1977; Weinberg, Gould Yukelson y Jackson, 1981, Bandura y Cervone, 1986; Schunk, 1995, Weigand y Stockham, 2000). El estudio de la Teoría de la Autoeficacia nace en 1977, por parte del Dr. Albert Bandura quien, por varios años, junto con otros colegas del campo de la psicología, la fue definiendo de manera más aplicada.

Según el origen del término "autoeficacia", esta es una palabra compuesta: "auto" que, de acuerdo con la Real Academia de la Lengua Española (2001), significa "propio" o "por uno mismo", por su parte, la palabra "eficacia", del latín efficaîa, se refiere a la capacidad de lograr el efecto deseado o esperado.

Bandura (1977), en su primer acercamiento, se refiere a la autoeficacia como "la creencia en la capacidad propia que tiene la persona, para organizar y realizar las acciones requeridas para producir los resultados deseados". Casi diez años después, Bandura y Cervone (1986) amplían el concepto y lo entienden como la seguridad que puede tener un individuo en sus propias habilidades, para solucionar una dificultad específica, en este sentido, el nivel de autoeficacia que posea una persona se convierte en una competencia deseable que se debe desarrollar en cualquier ámbito.

En el transcurrir de estos diez años (1977-1987), otros autores también han analizado y dado sus aportes en la comprensión del constructo. Weinberg, Gould Yukelson y Jackson (1981) han definido la autoeficacia como la certeza que tiene una persona de poder ejecutar satisfactoriamente determinada tarea. En el contexto de las Ciencias del Movimiento 
Humano, estas definiciones nos permiten entender la autoeficacia como una característica o competencia deseable en las personas, ya que si estas tienen un nivel alto de autoeficacia y conocen o han experimentado los beneficios del ejercicio, se espera que se les facilite la toma de decisiones de iniciar un programa de ejercicio físico o deportivo, a pesar de las ocupaciones y de las múltiples tareas por realizar en la vida tan vertiginosa que hoy tienen.

\section{Autoeficacia en la Actividad Física}

Diferentes investigaciones han determinado, en primera instancia, que la autoeficacia influye sobre el grado de motivación y compromiso para hacer ejercicio. Tal es el caso de Olivary y Urra (2007) quienes en su revisión de literatura indican que las percepciones de autoeficacia juegan un rol significativo en la conducta de hacer ejercicio, de manera que, las personas con mayores niveles de autoeficacia se comprometen con mayor frecuencia en un programa regular de actividad física que aquellos con niveles más bajos de autoeficacia (Rimal, 2001).

De igual forma, Astudillo-García y Rojas-Russell (2006) encontraron en una muestra de 199 estudiantes universitarios físicamente activos, una asociación positiva significativa entre el nivel de actividad física y la autoeficacia, es decir, a mayor actividad física semanal, mayor nivel de autoeficacia.

Por otra parte, algunas las investigaciones realizadas con el objetivo de determinar si la realización de un programa de ejercicios modifica la percepción de autoeficacia. En este sentido, Lockwood y Wohl (2012) encontraron que 71 estudiantes universitarios matriculados durante 15 semanas en un curso de bienestar de vida mejoraron no solo su condición física y nutrición, sino también su autoeficacia.

Por último, están los estudios que pretenden determinar si la autoeficacia juega un rol importante en el nivel de motivación o compromiso por continuar realizando ejercicio, ya que, según Bandura (1977), un nivel alto de autoeficacia puede influir directamente sobre la conducta futura. En este sentido, los resultados son bastante positivos. Tal es el caso de un estudio realizado con estudiantes universitarios de distintas Universidades de Hong Kong y Australia ( $n=1709$ ) en el cual se reporta que la autoeficacia es un fuerte determinante en la motivación para participar en actividades de recreación activa (Hiu-Lun y Coleman, 2009).

Por su parte, Bo y Xu (2008) reportan que, en un grupo de 218 estudiantes de una Universidad Pública en Kansas-EE.UU., la autoeficacia tuvo un impacto significativo en las 
motivaciones para involucrarse en ejercicio recreativo tanto en hombres como en mujeres, aun cuando las motivaciones en los varones se fundamentaban en el componente psicológico y social (i.e., reconocimiento social, competición) mientras que en las mujeres cobraba más interés el componente físico (i.e., bajar de peso y lucir mejor su cuerpo).

Con base en estos hallazgos, se planteó realizar la siguiente investigación con estudiantes universitarios que asistieron regularmente al curso de actividad deportiva impartido durante el verano de 2009. Este es un curso que forma parte del plan curricular para todas las carreras que ofrece la Universidad de Costa Rica, cuyo fin es promover un ambiente que estimule en la población estudiantil adoptar un estilo de vida activo.

Los propósitos de la investigación fueron: (1) comprobar si existe relación significativa entre el nivel de autoeficacia y la cantidad de actividad física que reporta el estudiantado al inicio del curso; (2) establecer si existe interacción significativa entre las mediciones efectuadas antes del curso y después de él y entre el tipo de actividad física (acondicionamiento físico y deporte) y el género, influenciando la autoeficacia.

\section{Metodología}

\subsection{Diseño}

El diseño utilizado en este estudio fue pre experimental, en el cual se realizó una medición anterior (pre-test) y posterior (post-test) de la variable autoeficacia a cada grupo de actividad deportiva (Campbell y Stanley, 1963).

\subsection{La muestra}

La población que se tomó en cuenta para el estudio fue la totalidad de estudiantes matriculados en el Curso de Actividad Deportiva que imparte la Escuela de Educación Física y Deportes de la Universidad de Costa Rica durante el III ciclo lectivo del 2009. El análisis se realizó con los 211 estudiantes que completaron los instrumentos de medición al inicio y al finalizar el curso.

\subsection{Instrumentos de medición}

El nivel de actividad física se estimó por medio del Cuestionario Internacional de Actividad Física (IPAQ, por sus siglas en inglés) (Craig y col., 2003). Este instrumento mide el grado de actividad física moderada, vigorosa y de actividades sedentarias. La validez y 
confiabilidad se ha obtenido para muestras de personas de 12 países en 6 continentes, en donde la confiabilidad para todas las versiones del IPAQ arrojan un coeficiente de correlación de Spearman Rho $=0.8$, cuando el instrumento se autoadministra o cuando se realiza por medio de una entrevista telefónica a personas entre los 18 y 69 años. La validez de criterio se ha reportado en $r=0.30$ al compararlo con acelerómetros para actividades moderadas, vigorosas, caminar y para comportamientos sedentarios.

La autoeficacia, para efectos del estudio, se entenderá como la confianza en sí mismo que tiene el/la estudiante para iniciar un plan de ejercicio así como para no dejarlo, según la definición de Bandura (1977). La escala que se utilizó para medirla fue la llamada "Encuesta de Autoeficacia para Comportamientos de Ejercicio" de Sallis, Pinski, Grossman, Patterson y Nader (1988). El cuestionario consiste en 12 ítems, 6 de ellos se refieren a la autoeficacia para hacer ejercicio y los otros 6 a la autoeficacia para no dejar de hacer ejercicio. Ambas sub-escalas tienen una confiabilidad "test-retest" de 0.68 y una consistencia interna de 0.83 y 0.85. Una correlación significativa entre el constructo de autoeficacia y el ejercicio vigoroso comprobó la validez del instrumento.

\subsection{Procedimientos}

Se explicó a la población estudiantil el propósito del estudio y se les pidió su colaboración voluntaria para quienes estuvieran interesados en participar. A quienes aceptaron colaborar se les repartieron los instrumentos escritos que debían llenar (i.e., IPAQ y el de autoeficacia) y después se les entregó un formulario de información general en el que se recopiló la edad, el sexo y el número de carné del participante, así como el grupo de actividad deportiva matriculado. De igual forma, todos los estudiantes fueron valorados en peso, talla y se determinó el Índice de Masa Corporal. Finalizadas las ocho semanas del curso se les aplicó de nuevo el cuestionario de autoeficacia.

\subsection{Ejercicio físico}

La persona responsable de cada grupo de actividad deportiva realizó una rutina de trabajo según la naturaleza de cada curso, es decir, los grupos de acondicionamiento físico realizaron rutinas de ejercicio aeróbico, anaeróbico y ejercicio contra resistencia o de pesas, por su parte, los cursos deportivos realizaron esencialmente rutinas específicas de los respectivos deportes, para este estudio, fútbol sala y taek-wondo. Cada clase incluía una 
presentación de las actividades u objetivo de la clase, seguida de un calentamiento, el desarrollo de la clase en sí (parte central de la clase) y, finalmente, actividades de relajación y de estiramiento para el regreso a un estado de reposo. Las actividades físicas y deportivas se realizaron durante 8 semanas, con una frecuencia de dos veces por semana, cada sesión tuvo una duración de 100 minutos.

\subsection{Análisis estadísticos}

En la estadística descriptiva se obtuvieron los valores mínimos, máximos, promedios y desviaciones estándar (DE) de las variables investigadas.

En la estadística inferencial se procedió a realizar una correlación de Pearson entre la autoeficacia y la actividad física reportada por los estudiantes al inicio del curso. Posteriormente, se realizaron dos ANOVAS de 3 vías (2 tipos de actividad física $\times 2$ géneros x 2 mediciones) para determinar el efecto de la actividad deportiva sobre: (1) la autoeficacia para hacer ejercicio (2) la autoeficacia para no dejar de hacer ejercicio.

Los datos de los gráficos se reportan como promedios \pm error estándar de la muestra (EEM).

Los análisis fueron realizados con el Paquete Estadístico para las Ciencias Sociales (SPSS Inc. Chicago, Illinois, USA) versión 15.0. Los valores de $p<0.05$ fueron aceptados como significativos.

\section{Análisis y Resultados de la Investigación}

En este apartado, se presentará la estadística descriptiva y luego, los resultados y análisis para la variable "autoeficacia para hacer ejercicio"; finalmente, los resultados y análisis para la variable "autoeficacia para no dejar de hacer ejercicio".

En el estudio participaron 211 estudiantes ( $n=108$ mujeres y $n=103$ hombres) matriculados en cinco diferentes modalidades de actividad deportiva impartidos por la Escuela de Educación Física y Deportes: a) Sistemas de preparación física principiante mixto, b) Taekwondo principiante mixto, c) Halterofilia principiante mixto, d) Fútbol salón principiante hombres y e) Fútbol salón principiante mujeres. Con estas modalidades se construyeron dos categorías que representaran fielmente la finalidad del curso: a) acondicionamiento físico $(n=103)$ y b) actividad deportiva $(n=108)$. 
En la Tabla 1 se puede apreciar la estadística descriptiva de las variables antropométricas de los 211 participantes.

Tabla 1

Características físicas de los participantes

\begin{tabular}{|l|c|c|c|}
\hline \multicolumn{1}{|c|}{ Variable } & Promedio $\pm \mathrm{DE}$ & Mínimo & Máximo \\
\hline Edad $(\mathrm{años})$ & $20.45 \pm 2.3$ & 18 & 37 \\
\hline Talla $(\mathrm{cm})$ & $165.37 \pm 8.8$ & 147 & 191 \\
\hline Peso $(\mathrm{kg})$ & $63.86 \pm 14.1$ & 35.85 & 124.8 \\
\hline Índice de Masa Corporal (IMC) & $23.25 \pm 4.3$ & 14.73 & 41.96 \\
\hline
\end{tabular}

Fuente: Campos, Cinthya y Solera, Andrea (2013). Resultados de las mediciones antropométricas realizadas a los estudiantes de actividad deportiva del verano 2009.

De la Tabla 1 se puede decir, en general, que el Índice de masa corporal promedio presenta valores de peso normal para la población estudiada.

Con el objetivo de comprobar si existe relación significativa entre el nivel de autoeficacia y la cantidad de actividad física que reporta la población estudiantil al inicio del curso, se calculó el Coeficiente de Correlación de Pearson, el valor obtenido fue de 0.42 $(p<0.001)$. La ecuación de regresión lineal fue $y=1.26 x+0.06$ (en donde $y=$ nivel de actividad física y $\mathrm{x}=$ nivel de autoeficacia). La ecuación de regresión lineal fue $\mathrm{y}=8,80 \mathrm{x}+$ 0,42 (en donde $y=$ nivel de actividad física y $x=$ nivel de autoeficacia). El error estándar de la estimación fue de 16,65 y el $\mathrm{R}^{2}$ de 0,176.

En el Gráfico 1 se presentan los resultados de la correlación de Pearson con la línea de regresión de mejor ajuste, en los cuales se puede detallar que, a mayor nivel de actividad física, los estudiantes presentan un mayor nivel de autoeficacia.

Los resultados del Gráfico 1 concuerdan con los reportados por Astudillo-García y Rojas-Russell (2006) quienes realizaron un estudio con 199 estudiantes universitarios físicamente activos de la Facultad de Psicología en la Universidad de Zaragoza, México. El estudio fue de corte descriptivo, se aplicaron cuestionarios para estimar el nivel de actividad física por semana, para conocer el nivel de autoeficacia al ejercicio y para conocer la actitud al cambio para iniciar un programa de ejercicio físico. El análisis bivariado mostró una asociación significativa entre el nivel de actividad física y la autoeficacia y, además, entre la actividad física y la disposición al cambio para iniciar un programa de ejercicio físico.

Asimismo, Hiu-Liu y Coleman (2009) encontraron que la autoeficacia tiene relación significativa con el interés, la intención y la participación de estudiantes universitarios en 
actividades de recreación activa; participaron en este estudio 1709 estudiantes de distintas universidades de Hong Kong y Australia.

\section{Gráfico 1}

Correlación entre el nivel de actividad física por semana y la autoeficacia para hacer ejercicio al inicio del curso lectivo

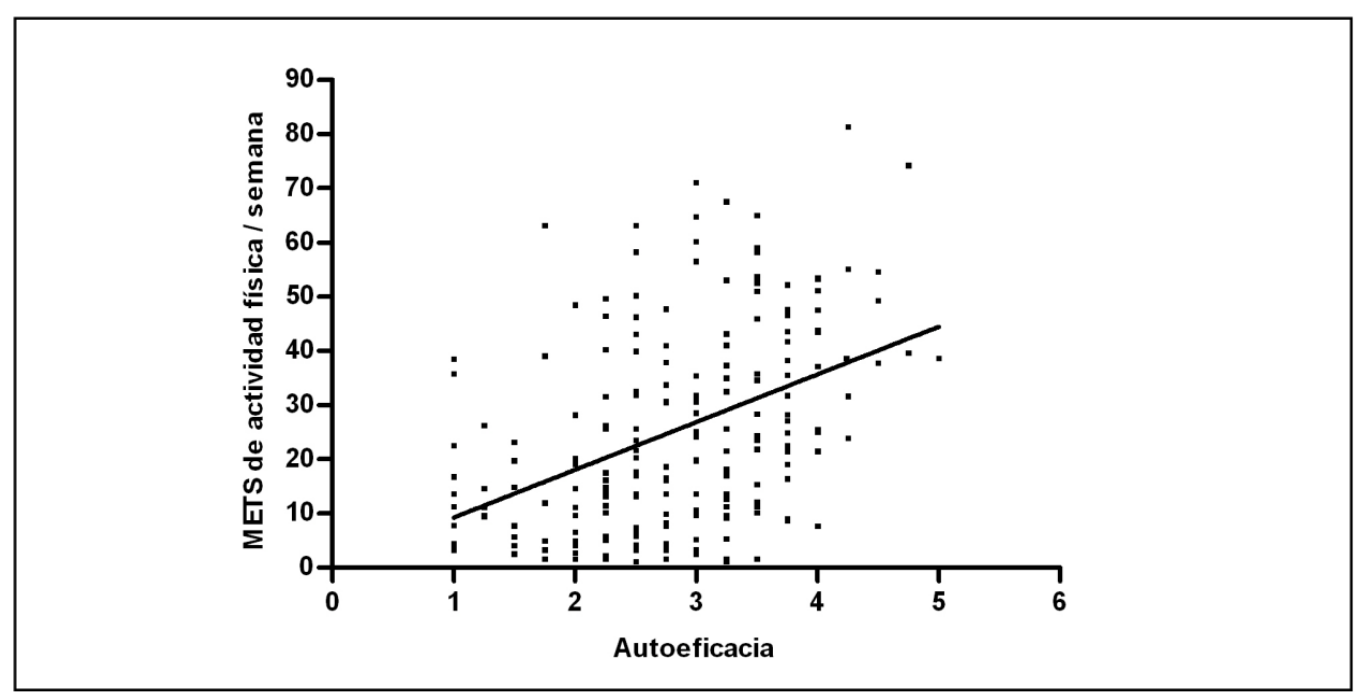

$r=0.42, p \leq 0.001$.

Fuente: Campos, Cinthya y Solera, Andrea (2013). Resultados de los cuestionarios de actividad física y autoeficacia aplicados al inicio y final del curso lectivo.

Otro estudio, en la misma línea, realizado por Bo y Xu, (2008) analiza la contribución de la autoeficacia en las motivaciones tanto psicológicas como interpersonales para realizar ejercicio, en estudiantes universitarios de China, Shangai. Los motivos psicológicos incluían factores como tensión, control, disfrute, desafío; mientras que los motivos interpersonales respondían a factores como reconocimiento social, afiliación y competición. Los resultados mostraron que el constructo autoeficacia es predictor de las motivaciones psicológicas (22\% de la varianza total) e interpersonales (18\% de la varianza total) para la mujeres y de las motivaciones interpersonales para los varones (20\% de la varianza total). En resumen, la autoeficacia, en forma generalizada, tiende a ser una variable que se asocia con el interés, la intención e incluso la participación en actividades físicas en la población universitaria.

En la Tabla 2 se puede apreciar la estadística descriptiva (promedios \pm DE) de la autoeficacia en sus dos variables de estudio, según el tipo de actividad física realizada, el género y el momento de la medición. 
Tabla 2

Estadística descriptiva de la autoeficacia según el tipo de actividad física, el género y la medición

\begin{tabular}{|c|c|c|c|c|c|}
\hline \multirow{2}{*}{ Autoeficacia } & Tipo de actividad física & \multicolumn{2}{|c|}{ Mujeres $(\mathrm{n}=108)$} & \multicolumn{2}{c|}{ Hombres $(\mathrm{n}=103)$} \\
\cline { 3 - 6 } & & Pre-test & Pos-test & Pre-test & Pos-test \\
\hline $\begin{array}{c}\text { Para hacer } \\
\text { ejercicio }\end{array}$ & $\begin{array}{c}\text { Acondicionamiento } \\
(\mathrm{n}=103)\end{array}$ & $2.7 \pm 0.9$ & $2.6 \pm 0.8$ & $3.3 \pm 0.6$ & $3.2 \pm 0.7$ \\
\cline { 2 - 6 } & $\begin{array}{c}\text { Deporte } \\
(\mathrm{n}=108)\end{array}$ & $2.4 \pm 0.9$ & $2.5 \pm 0.9$ & $2.9 \pm 0.8$ & $3.0 \pm 0.9$ \\
\hline $\begin{array}{c}\text { Para no dejar de } \\
\text { hacer ejercicio }\end{array}$ & $\begin{array}{c}\text { Acondicionamiento } \\
(\mathrm{n}=103)\end{array}$ & $2.7 \pm 0.9$ & $2.8 \pm 0.8$ & $3.3 \pm 0.7$ & $3.4 \pm 0.8$ \\
\cline { 2 - 6 } & $\begin{array}{c}\text { Deporte } \\
(\mathrm{n}=108)\end{array}$ & $2.7 \pm 1.0$ & $2.7 \pm 0.9$ & $3.3 \pm 0.7$ & $3.3 \pm 0.9$ \\
\hline
\end{tabular}

Fuente: Campos, Cinthya y Solera, Andrea (2013). Resultados de los cuestionarios de actividad física y autoeficacia aplicados al inicio y final del curso lectivo.

Con el propósito de conocer si hubo un cambio significativo en el nivel de autoeficacia debido a la actividad deportiva que realizaron los estudiantes durante el III ciclo lectivo, se realizó un análisis de varianza (ANOVA) de 3 vías según el tipo de actividad física, el género y la medición inicial y final de la autoeficacia.

Con respecto a la autoeficacia para hacer ejercicio, en la Tabla 3 se presentan los resultados del ANOVA de 3 vías (2 tipos de actividad física $\times 2$ géneros $\times 2$ mediciones). Como se puede apreciar, la autoeficacia para hacer ejercicio está significativamente influenciada por el tipo de actividad física y el género.

Tabla 3

Resultados de los ANOVAS de 3 vías de la autoeficacia para hacer ejercicio

\begin{tabular}{|c|l|c|c|}
\hline Autoeficacia & \multicolumn{1}{|c|}{ Variable } & F & $\mathbf{p} \leq$ \\
\hline \multirow{5}{*}{ Para hacer ejercicio } & Actividad Física & 4.42 & 0.04 \\
\cline { 2 - 4 } & Género & 26.04 & 0.001 \\
\cline { 2 - 4 } & Medición & 0.03 & 0.86 \\
\cline { 2 - 4 } & Actividad Física x Género & 0.00 & 0.99 \\
\cline { 2 - 4 } & Actividad Física x Medición & 1.93 & 0.17 \\
\cline { 2 - 4 } & Género x Medición & 0.02 & 0.90 \\
\cline { 2 - 4 } & Actividad Física x Medición x Género & 0.12 & 0.73 \\
\hline
\end{tabular}

Fuente: Campos, Cinthya y Solera, Andrea (2013). Resultados de los cuestionarios de actividad física y autoeficacia aplicados al inicio y final del curso lectivo.

En el Gráfico 2 se puede detallar que, indistintamente del género y de la medición, los participantes de los grupos de acondicionamiento físico mostraron mejor autoeficacia para hacer ejercicio que los participantes de los grupos de deportes $(p=0.04)$. Sin embargo, como las interacciones dobles para género y actividad física no son significativas, los resultados de 
la Tabla 3 indican que no es por efecto del tipo de ejercicio que este grupo de estudiantes presenta mayores de niveles de autoeficacia, sino que desde el inicio los estudiantes que escogieron los cursos de acondicionamiento físico presentaron niveles mayores de autoeficacia, lo que permite plantear la hipótesis o la interrogante de investigación acerca de cuáles serían las razones que influyeron para que esta tendencia se presentara.

\section{Gráfico 2}

Nivel de autoeficacia para hacer ejercicio según el tipo de actividad física realizada

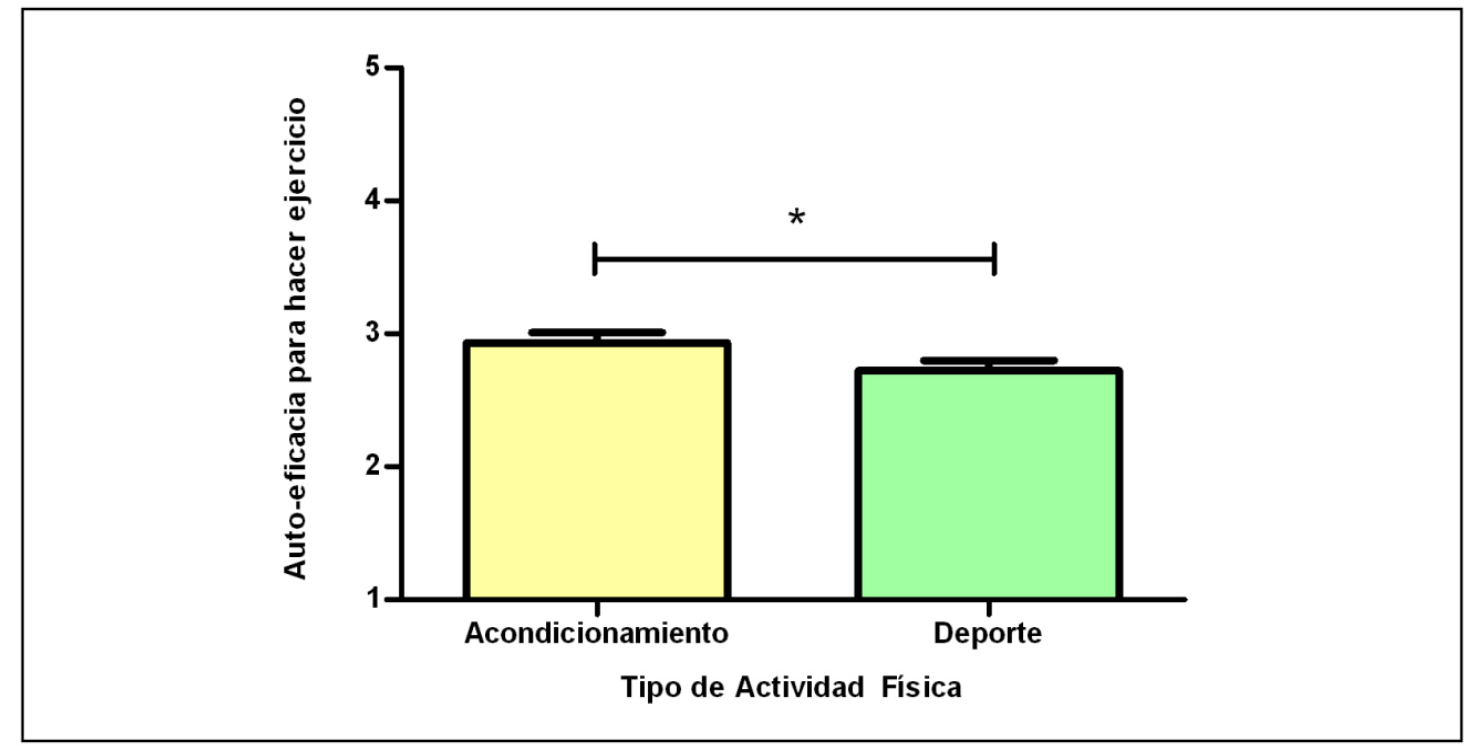

${ }^{*} p=0.04$. Datos se reportan como promedios \pm EEM

Fuente: Campos, Cinthya y Solera, Andrea (2013). Resultados de los cuestionarios de actividad física y autoeficacia aplicados al inicio y final del curso lectivo.

En relación con el género, en el Gráfico 3 se muestra que indistintamente de la medición o del tipo de actividad física, los puntajes de autoeficacia para hacer ejercicio son significativamente mayores en los hombres que en las mujeres $(p<0.001)$.

Igualmente, en este caso, ninguna interacción doble es significativa (ver Tabla 3), lo que indica que, desde el inicio, las mujeres presentaron niveles inferiores de autoeficacia y esta tendencia permanece hasta el final del curso de la "actividad deportiva". Este resultado es confirmado por otros autores como Hernández-Álvarez, Velásquez-Buendía, MartínezOrroño, Garoz-Puerta y Tejero (2011), quienes en un estudio descriptivo realizado con 1288 jóvenes, de seis regiones españolas, reportan que las adolescentes presentan niveles inferiores de autoeficacia motora que los varones. 
Gráfico 3

Nivel de autoeficacia para hacer ejercicio según el género

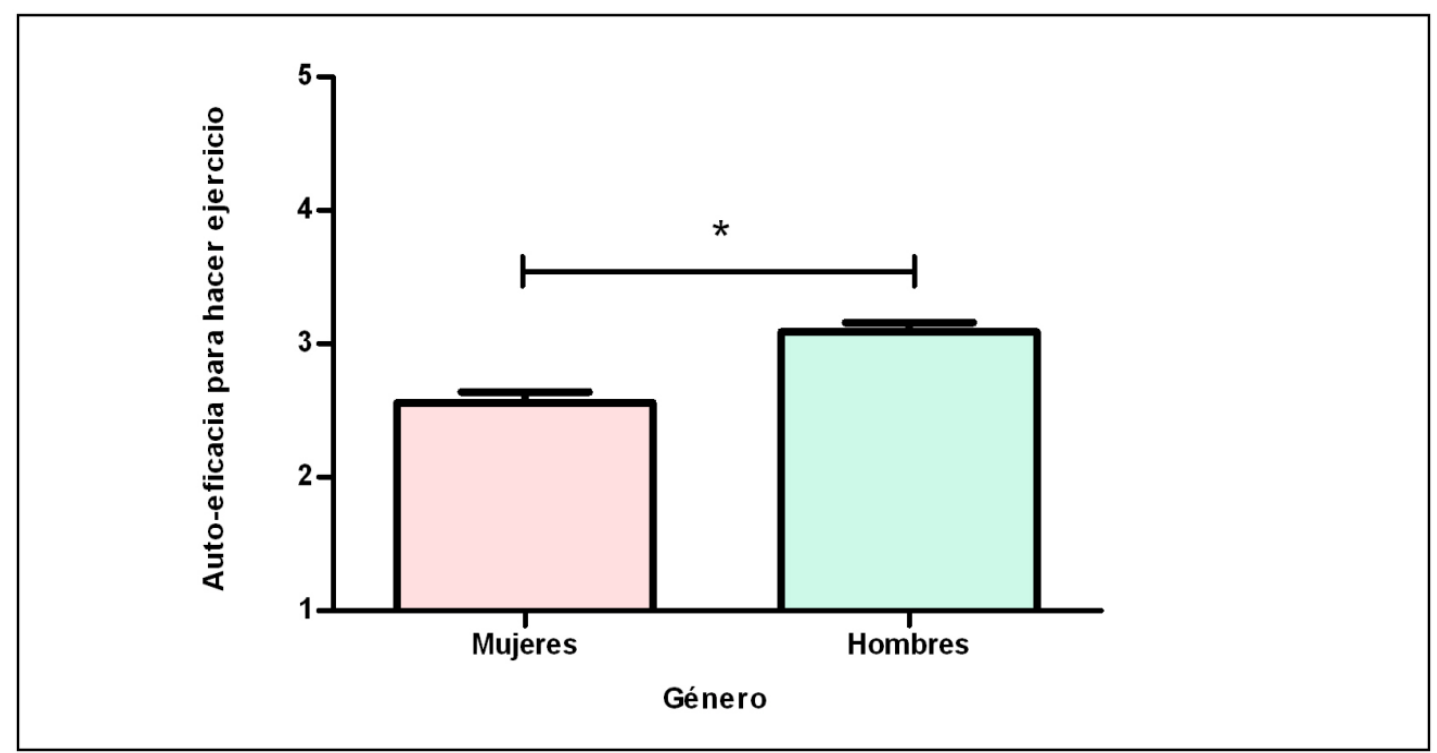

${ }^{*} \mathrm{p}<0.001$. Datos se reportan como promedios \pm EEM

Fuente: Campos, Cinthya y Solera, Andrea (2013). Resultados de los cuestionarios de actividad física y autoeficacia aplicados al inicio y final del curso lectivo.

Asimismo, Astudillo-García y Rojas-Russell (2006) reportan niveles inferiores de autoeficacia para hacer ejercicios en mujeres universitarias que en varones de edad similar, cuando se trata de enfrentar situaciones que involucran emociones, no tener tiempo, o padecimientos. En este sentido, resulta indudable la necesidad de indagar las razones por las que las mujeres poseen menor autoeficacia y plantear formas o estrategias que permitan fortalecerla.

Según Gordon-Larsen, McMurray y Popkin (2000), las clases de Educación Física proveen oportunidades importantes para promover comportamientos de salud extracurriculares y patrones de actividad que alargan la vida. En la misma línea, Jackson, Myers, Taylor y Beauchamp (2012), encontraron que los estudiantes confiaban más en sus propias capacidades si percibían que su instructor también lo hacía; Lent y Lopez (2002), por su parte, mencionan que cuando una persona cree que sus capacidades son vistas favorablemente por su instructor, esta se siente apoyada, lo que le permite reforzar sus acciones en el dominio. De acuerdo con estas recomendaciones, si desde la etapa escolar, el educador físico o el entrenador logra fortalecer sus clases con expresiones positivas hacia las destrezas de sus alumnados o deportistas, es posible formar ciudadanos con mayor 
confianza en sí mismos al momento de enfrentar nuevas experiencias deportivas o de actividad física.

Con respecto a la autoeficacia para no dejar de hacer ejercicio, en la Tabla 4 se presentan los resultados del ANOVA de 3 vías (2 tipos de actividad física $\times 2$ géneros $\times 2$ mediciones). Como se puede notar, dicha autoeficacia se ve influenciada tanto por el género como por el momento de la medición.

Tabla 4

Resultados de los ANOVAS de 3 vías de la autoeficacia para no dejar de hacer ejercicio

\begin{tabular}{|c|l|c|c|}
\hline Autoeficacia & \multicolumn{1}{|c|}{ Variable } & $\mathbf{F}$ & $\mathbf{p} \leq$ \\
\hline \multirow{4}{*}{$\begin{array}{c}\text { Para no dejar de } \\
\text { hacer ejercicio }\end{array}$} & Actividad Física & 0.31 & 0.58 \\
\cline { 2 - 4 } & Género & 19.35 & 0.001 \\
\cline { 2 - 4 } & Medición & 5.6 & 0.02 \\
\cline { 2 - 4 } & Actividad Física x Género & 0.07 & 0.80 \\
\cline { 2 - 4 } & Actividad Física x Medición & 0.18 & 0.68 \\
\cline { 2 - 4 } & Género x Medición & 0.08 & 0.78 \\
\cline { 2 - 4 } & Actividad Física x Medición x Género & 0.44 & 0.51 \\
\hline
\end{tabular}

Fuente: Campos, Cinthya y Solera, Andrea (2013). Resultados de los cuestionarios de actividad física y autoeficacia aplicados al inicio y final del curso lectivo.

En el Gráfico 4 se puede apreciar cómo indistintamente del tipo de actividad física y del momento de la medición, la autoeficacia para no dejar de hacer ejercicio fue mayor en los hombres que en las mujeres $(p<0.001)$. En este caso, la estadística muestra el mismo comportamiento que la autoeficacia para hacer ejercicio, es decir, desde el inicio las mujeres presentan niveles inferiores de autoeficacia y esta tendencia se mantiene una vez terminado el ciclo lectivo.

Por último, en el Gráfico 5 se puede apreciar que indistintamente del tipo de ejercicio y del género, la autoeficacia para no dejar de hacer ejercicio tuvo un aumento significativo entre la medición al inicio del curso y la medición al finalizar el curso ( $p=0.02)$. 


\section{Gráfico 4}

Nivel de autoeficacia para no dejar de hacer ejercicio según el género

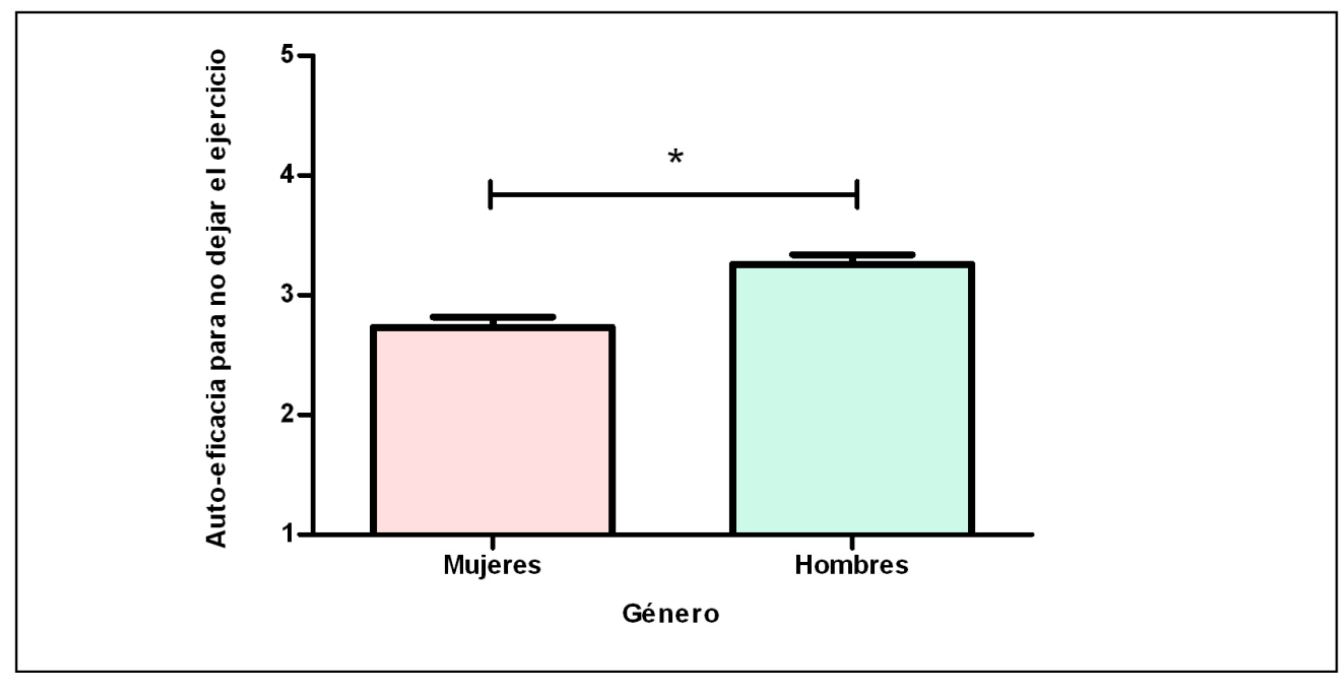

${ }^{*} p<0.001$. Datos se reportan como promedios \pm EEM

Fuente: Campos, Cinthya y Solera, Andrea (2013). Resultados de los cuestionarios de actividad física y autoeficacia aplicados al inicio y final del curso lectivo.

\section{Gráfico 5}

Nivel de autoeficacia para no dejar de hacer ejercicio según el momento de la medición

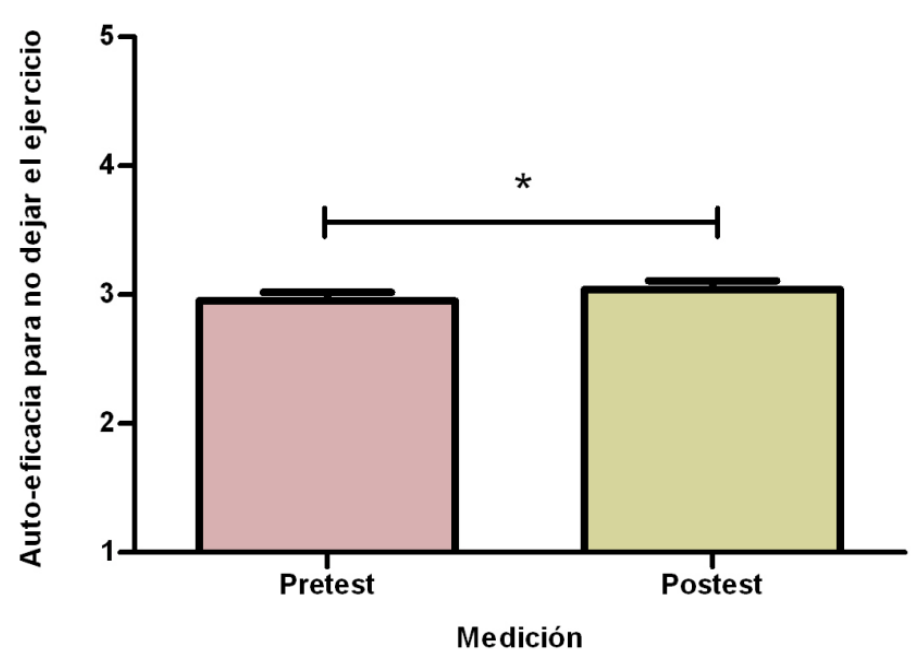

${ }^{*} \mathrm{p}=0.02$. Datos se reportan como promedios \pm EEM

Fuente: Campos, Cinthya y Solera, Andrea (2013). Resultados de los cuestionarios de actividad física y autoeficacia aplicados al inicio y final del curso lectivo. 
De acuerdo con el Gráfico 5, se presenta una mejoría significativa en la autoeficacia para no dejar de hacer ejercicio una vez finalizado el curso de actividad deportiva, es decir, el participar de un curso de este tipo beneficia el perfil del estudiantado para mantener su nivel de actividad física, ya que su nivel de confianza en sí mismo para no dejar de hacer ejercicio aumenta significativamente. Lockwood y Wohl (2012) realizaron un estudio similar al nuestro ya que analizaron el efecto del ejercicio en la autoeficacia en estudiantes universitarios que matricularon un curso teórico práctico denominado "Bienestar duradero". El curso comprendía sesiones prácticas de actividad física y charlas acerca del buen cuidado personal. Los resultados mostraron un aumento significativo en la autoeficacia al ejercicio una vez finalizado el curso lectivo.

Otra publicación reciente es la de Tayama, Yamasaki, Tamai, Hayashida, y Shirabe, (2012) quienes encontraron que, a mayor nivel de autoeficacia, mayor nivel de actividad física. Este hallazgo fue comprobado al constatar que los estudiantes universitarios con mayor nivel de autoeficacia al ejercicio realizaron mayor ejercicio semanal que quienes tenían menor nivel de la misma.

Estos hallazgos son muy importantes ya que es clara la relación que existe entre el nivel de autoeficacia y el nivel de actividad física en la población estudiantil, incluso adolescente. Dos estudios, también recientes, realizados con adolescentes, presentan resultados similares. Spence y col. (2010) con una muestra de 4779 adolescentes con una edad promedio de 13.64 años reportan que existe una correlación fuerte entre autoeficacia y actividad física en mujeres, no obstante, los niveles de actividad son mayores en los niños que en las niñas debido a que presentan mayores niveles de autoeficacia al ejercicio. Por su parte, Inchley, Kirby y Currie (2011), al medir 641 adolescentes, determinan que las niñas con mayores índices de autoeficacia aumentan la probabilidad de hacer ejercicio en el futuro mientras que los niños aumentan su probabilidad en la medida que se perciben físicamente más hábiles para el deporte.

\section{Conclusiones}

Promover espacios para la práctica de ejercicio o la actividad física se ha convertido en una tarea de las instituciones de educación formal, empresas públicas y privadas e incluso en espacios familiares con el fin de estimular el organismo para un mejor desempeño físico y psicológico. 
La autoeficacia para hacer ejercicio y para no dejar de hacer ejercicio es una característica deseable en los tiempos actuales en los cuales la agenda diaria está llena de actividades que tienden prioridazarse a la práctica de ejercicio o actividad física.

En el análisis de los resultados, tanto para comprobar si los niveles de actividad física están relacionados con el nivel de autoeficacia del estudiantado como para determinar si los cursos de actividad deportiva ofrecidos podían modificar los niveles de autoeficacia para hacer ejercicio y los niveles para no dejar de hacer ejercicio se evidencia que:

- A mayor nivel de actividad física la población estudiantil del estudio presenta un mayor nivel de autoeficacia para hacer ejercicio.

- El estudiantado que seleccionó el curso de acondicionamiento físico desde el inicio presentó niveles superiores de autoeficacia para hacer ejercicio que los estudiantes que matricularon cursos de deportes.

- En comparación con los varones, las mujeres presentan niveles inferiores de autoeficacia, tanto para hacer ejercicio como para no dejar de hacerlo. Esta situación se presenta por igual al inicio y al final del curso de actividad física o deportiva.

- El estudiantado presentó un aumento significativo en el nivel de autoeficacia para no dejar de hacer ejercicio, independientemente del género y el tipo de actividad física, una vez finalizado el curso de actividad física o deportiva.

Podemos decir que los cursos de actividad deportiva que ofrece la Universidad de Costa Rica para toda la población estudiantil tiene efectos positivos en la autoeficacia para no dejar de hacer ejercicio, sin embargo, falta por investigar por qué las mujeres tienen menores niveles de autoeficacia hacia el ejercicio que los varones y si estos efectos encontrados en un curso de verano se mantienen para los cursos que se ofrecen en los ciclos regulares cuando la frecuencia del curso es solamente de una vez por semana.

\section{Referencias}

Astudillo-García, Claudia I. y Rojas-Russell, Mario E. (2006). Auto eficacia y disposición al cambio para la realización de actividad física en estudiantes universitarios. Acta Colombiana de Psicología, 9(1), 41-49.

Bandura, Alberth y Cervone, Daniel. (1986). Differential engagement of self-reactive influences in cognitve motivation. Organizational Behavior \& Human Decision Process, 38(1), 92-113. doi: 10.1016/0749-5978(86)90028-2 
Bandura, Alberth. (1977). Self-efficacy: toward a unifying theory of behavioral change. Psycological Review, 84,191-215.

Bo, Shen y Xu Chiren. (2008). Effects of self-efficacy, body mass and cardiorespiratory fitness on exercise motives in chinese collage students. Journal of Physical Activity and Health, 5, 706-718.

Campbell, Donald T. y Stanley, Julian C. (2001). Experimental and quasi-experimental designs for research [Diseños experimentales y cuasi experimentales de investigación] (8 ed.). Buenos Aires: Amorrortu Editors S.A.

Campos, Cinthya y Moncada José. (octubre, 2009). El efecto de diversas actividades físicas y deportivas en el estado de ánimo depresivo en estudiantes universitarios costarricenses. Ponencia presentada en el XVI Simposio Internacional en Ciencias del Deporte, el Ejercicio y la Salud, San José, Costa Rica.

Campos, Cinthya y Moncada, José. (2012). Efecto de diversas actividades físicas en el estado anímico depresivo en estudiantes universitarios costarricenses. Revista Costarricense de Salud Pública, 21(1), 9-14.

Craig, Cora L., Marshall, Alison L., Sjöström, Michael, Bauman, Adrian E., Booth, Michael L., Ainsworth, Barbara E., Pratt, Michael, Ekelund, Ulf., Yngve, Agneta., Sallis, James F. y Oja, Pekka. (2003). International physical activity questionnaire (IPAQ): 12-country reliability and validity. Medicine \& Science in Sports \& Exercise, 35(8), 1381-1395.

Gordon-Larsen, Penny, McMurray, Robert G. y Popkin Barry M. (2000). Determinants of adolescent physical activity and inactivity pattern. Pediatrics, 105, 1-8. doi: 10.1542/peds.105.6.e83

Hernández-Álvarez, Juan L., Velásquez-Buendía, Roberto, Martínez-Gorroño, María E., Garoz-Puerta, I. y Tejero, Carlos M. (2011). Escala de auto-eficacia motriz: propiedades psicométricas y resultados de su aplicación a la población escolar española. Revista de Psicología del Deporte, 20(1), 13-28.

Hiu-Lun Tsai, Eva y Coleman, Denis. (2009). The influence of constraints and self-eficacies on participation in regular active recreation. Leisure Sciences, 31, 364-383. doi: 10.1080/01490400902988309

Inchley, Jo, Kirby, Jo y Currie, Candace. (2011). Longitudinal changes in physical selfperceptions and associations with physical activity during adolescence. Pediatric Exercise Sciences, 23, 237-249.

Jackson, Ben, Myers, Nicholas D., Taylor, lan M. y Beauchamp Mark R. (2012). Relational efficacy beliefs in physical activity classes: a test of the tripartite model. Journal of Sport \& Exercise Psychology, 34, 285-304.

Legrand, Fabien y Philippe, Jean. (2007). Antidepressant effects associated with different exercise conditions in participants with depression: a pilot study. Journal of Sport \& Exercise Psychology, 29, 348-364. 
Lent, Robert W. y López, Frederick G. (2002). Cognitive ties that bind: A tripartite view of efficacy beliefs in growth-promoting relationships. Journal of Social and Clinical Psychology, 21, 256-286. doi: 10.1521/jscp.21.3.256.22535

Lockwood, Park y Wohl, Roy. (2012). The Impact of a 15-week lifetime wellness course on behavior change and self-efficacy in college students. College Students Journal, 46(3), 628-641.

Olivary Medina, Cecilia y Urra Medina, Eugenia. (2007). Autoeficacia y conductas de salud. Ciencia y Enfermería, 13(1), 9-15.

Real Academia Española. (2001). Diccionario de la Lengua Española. (22, Tomo 1) Madrid, España, Espasa Calpe.

Rethorst, Chad, Wipfli, Bradley y Landers, Daniel. (2009). The antidepressive effects of exercise. A Meta-Analysis of Randomized Trials. Sports Medicine, 39(6), 491-511.

Rimal, Rajiv. (2001). Longitudinal influences of knowledge and self-efficacy on exercise behavior: test of a mutual reinforcement model. Journal of Health Psychology, 6(1), 3146.

Sallis, James F., Pinski, Robin B., Grossman, Robin M., Patterson, Thomas L., y Nader, Philip R. (1988). The development of self-efficacy scales for health related diet and exercise behaviors. Health Education Research, 3(3), 283-292.

Schunk, Dale. (1995). Self-efficacy, motivation and performance. Journal of Applied Sport Psycology, 7, 112-137.

Spence, John C., Blanchard, Chris M., Clark, Marianne, Plotnikoff, Ronald C., Storey, Kate E. y McCargar, Linda. (2010). The role of self-efficacy in explaining gender differences in physical activity among adolescents. A multilevel analysis. Journal of Physical Activity \& Health, 7(2), 176-183.

Tayama, Yun, Yamasaki, Hironori, Tamai, Mami, Hayashida, Msaki y Shirabe, Susumu (2012). Effect of baseline self-efficacy on physical activity and psychological stress after a one-week pedometer intervention. Perceptual and Motor Skills, 114(2), 407-418.

Weigand, Daniel y Stockham Kimberley. (2000). The importance of analyzing positionspecific self-efficacy. Journal of Sport Behavior 23(1), 61-69. Recuperado de http://www.questia.com/read/1G1-61909298/the-importance-of-analyzing-positionspecific-self-efficacy

Weinberg, Robert, Gould, Daniel, Yukelson, David, y Jackson, Allen. (1981). The effect of preexisting and manipulated self-efficacy on a competitive muscular endurance task. Journal of Sport Psychology, 3(4), 345-354. 